INDO GLOBAL JOURNAL OF

PHARMACEUTICAL SCIENCES

ISSN 2249- 1023

\title{
An Overview of Antioxidant Properties of Probiotics
}

\author{
Kritika Sehgal, Hitesh Shetty, Akshita Gupta, Shahrukh Husain, Smriti Gaur * \\ Department of Biotechnology, Jaypee Institute of Information Technlogy, Noida, Uttar Pradesh, India
}

Address for Correspondance: Smriti Gaur, smriti.gaur@jiiit.ac.in

\begin{abstract}
Keywords
Probiotics;

Antioxidants;

Antioxidant Assays;

Lactic Acid

Bacteria (LAB);

Irritable Bowel

Syndrome.
\end{abstract}

\begin{abstract}
A number of Lactobacillus species, Bifidobacterium species, Saccharomyces boulardii, and other microbes have been characterized and used as probiotic strains. These strains are live microorganisms used as food supplements to confer health benefits, and may be used with synbiotics or prebiotics. The health benefits range from bowel activity regulation to exerting antagonistic effect on the gastroenteric pathogens like Clostridium difficile, Campylobacter jejuni, Helicobacter pylori and rotavirus, thus neutralizing food mutagens produced in colon, thereby lessening allergic reactions, lowering serum cholesterol and antioxidant activity. Antioxidants prevent cell damage from free radicals; the oxidative damage caused due to free radicals has been responsible for diseases such as Alzheimer's, Irritable Bowel Syndrome, cancer \& diabetes, among others. While our body does possess antioxidants, such as catalase \& superoxide dismutase, there is a need to supplement our body with additional sources of antioxidants. This is where probiotics come in. Probiotics have been found to possess antioxidant activity, which can be measured by Reactive Oxygen Species (ROS) scavenging, metal ion chelation, enzyme inhibition, and reduction and inhibition of ascorbate autoxidation. DPPH assay, Hydrogen peroxide scavenging assay, ABTS assay, beta carotene linoleic acid method, metal chelating activity, phosphomolybdenum method are some of the commonly used antioxidant assays. DPPH method has maximum application in vivo while ethanol has been used with the highest frequency as a solvent for extraction purpose. Study of probiotics from various industries has been done. (C) 2016 iGlobal Research and Publishing Foundation. All rights reserved.
\end{abstract}

Conference Proceedings: International Conference on Advances in Plant and Microbial Biotechnology (PMB2017); JIIT, Noida: February 02-04, 2017

Indo Global Journal of Pharmaceutical Sciences( ISSN 22491023 ; CODEN- IGJPAI; NLM ID: 101610675) indexed and abstracted in EMBASE(Elsevier), SCIRUS(Elsevier),CABI, CAB Abstracts, Chemical Abstract Services(CAS), American Chemical Society(ACS), Index Copernicus, EBSCO, DOAJ, Google Scholar and many more. For further details, visit http://iglobaljournal.com 